Théologiques

Théologiques

\title{
Le travail aux prises avec le néo-libéralisme économique
}

\section{Michel Beaudin}

Volume 3, numéro 2, octobre 1995

Crise du travail, crise de civilisation

URI : https://id.erudit.org/iderudit/602421ar

DOI : https://doi.org/10.7202/602421ar

Aller au sommaire du numéro

\section{Éditeur(s)}

Faculté de théologie de l'Université de Montréal

\section{ISSN}

1188-7109 (imprimé)

1492-1413 (numérique)

Découvrir la revue

\section{Citer cet article}

Beaudin, M. (1995). Le travail aux prises avec le néo-libéralisme économique. Théologiques, 3(2), 13-32. https://doi.org/10.7202/602421ar

\section{Résumé de l'article}

Qu'arrive-t-il au travail? Dans quel tourbillon économique et politique est-il entraîné? Le diagnostic sur le processus qui donne lieu à tant de drames personnels et à un véritable séisme social commence par un relevé de données et de tendances touchant l'évolution récente du travail : tertiarisation, raréfaction, dualisation et précarisation, " tiersmondialisation ", et dégradation de ses conditions d'exercice. Il se poursuit ensuite par l'examen du paradoxe du découplage de la croissance et de l'emploi, paradoxe dont la logique appartient au modèle néo-libéral, dernière en date des figures du capitalisme au XX ${ }^{\mathrm{e}}$ siècle. Enfin, le travail est abordé sous l'angle de sa dépendance de l'" economically correct ", à travers lequel le paradigme du marché s'auto-transcende et sacrifie les plus faibles dans la société.
Tous droits réservés @ Faculté de théologie de l'Université de Montréal, 1995
Ce document est protégé par la loi sur le droit d'auteur. L’utilisation des services d'Érudit (y compris la reproduction) est assujettie à sa politique d'utilisation que vous pouvez consulter en ligne.

https://apropos.erudit.org/fr/usagers/politique-dutilisation/ 


\title{
Le travail aux prises avec le néo-libéralisme économique
}

\author{
Michel BEAUDIN \\ Faculté de théologie \\ Université de Montréal
}

\section{RÉSUMÉ}

Qu'arrive-t-il au travail? Dans quel tourbillon économique et politique est-il entraîné? Le diagnostic sur le processus qui donne lieu à tant de drames personnels et à un véritable séisme social commence par un relevé de données et de tendances touchant l'évolution récente du travail: tertiarisation, raréfaction, dualisation et précarisation, «tiersmondialisation», et dégradation de ses conditions d'exercice. Il se poursuit ensuite par l'examen du paradoxe du découplage de la croissance et de l'emploi, paradoxe dont la logique appartient au modèle néo-libéral, dernière en date des figures du capitalisme au XXe siècle. Enfin, le travail est abordé sous l'angle de sa dépendance de l'«economically correct», à travers lequel le paradigme du marché s'auto-transcende et sacrifie les plus faibles dans la société.

Pour la majorité d'entre nous, nos conditions de vie dépendent d'un revenu, lié lui-même à un travail, lequel, dans une société fondée sur la propriété privée des moyens d'enrichissement économique et sur le contrat, prend une forme particulière appelée emploi, c'est-à-dire travail loué. C'est dire combien la question du travail est indissociable de celle de l'économie.

Quand on songe que non seulement nos besoins vitaux mais également notre reconnaissance sociale tiennent à un emploi décent, on peut imaginer sans peine l'ampleur personnelle et sociale de la secousse provoquée par la détérioration récente et apparemment inexorable de la situation de l'emploi. Que ce soit à travers les nouvelles répétées de «licenciements massifs ", la perte de son emploi par un parent ou un ami, ou encore à 
travers les propos et le regard de nos enfants où se lit une inquiétude paralysante face à l'avenir, et cela malgré les diplômes anticipés les plus solides, voilà autant d'indices qui nous font spontanément pressentir que c'est une société et peut-être même une civilisation qui craquent à travers la fracture de l'emploi.

D'autres exploreront le bien-fondé de ce pressentiment. Pour ma part, je me limiterai, ici, à décrire et à analyser le "comment " de la crise, espérant ainsi préciser l'objet de ce dossier. Je tenterai d'y arriver de trois façons: (1) en faisant état de quelques données et paradoxes sur ce phénomène; (2) en essayant de comprendre la singularité de la crise actuelle du travail par l'examen des trois modèles économico-politiques du capitalisme qui se sont succédés au $\mathrm{XX}^{\mathrm{e}}$ siècle; (3) enfin, en revenant sur certains traits de l'économie et de l'idéologie néo-libérales (l'economically correct) qui permettent de mieux saisir le caractère structurel de l'impasse touchant le travail. Je concluerai par l'énoncé de quelques questions ouvrant aux autres contributions de ce dossier.

\section{Données et paradoxes ou quand l'économie va bien, et les gens, mal}

Comment expliquer le "ça va mal a'shoppe" ou "ça va mal au bureau» du travailleur ou de la travailleuse rentrant à la maison le soir ? Où s'en va le travail? Comment se décide qui travaillera et quel travail il fera? Sans répéter des chiffres et des données abondamment diffusés ailleurs, on ne peut éviter d'évoquer ici les grandes tendances permettant de tracer un portrait sommaire de ce qui est en train d'arriver au travail, ne serait-ce que pour savoir de quoi il est question.

Des titres glanés récemment dans les journaux sont déjà extrêmement révélateurs des diverses facettes de la situation: "Séismes en vue sur le marché du travail "; "L'emploi est entré dans une nouvelle ère glaciaire "; "Le plein emploi, une illusion "; "Les mises à pied massives se poursuivront, prévoit Toffler »; "Monde du travail: dérapage incontrôlé »; "La crise de l'emploi: le choc d'une révolution industrielle "; "La job steady perd son emploi "; "Survivre "; "Le marché du travail aurait un "excès de travailleurs" "; "Pas de reprise pour les jeunes chômeurs "; "Détenteurs de doctorat et chômage "; " $50 \%$ des emplois créés au Canada depuis 1980 sont précaires et mal payés "; "Vers une société précaire "; "Maudits soient les bits"; "La mondialisation nous force à repenser l'emploi, selon la chambre de commerce "; "L'épuisement professionnel cause des maux de tête aux entreprises"; "Le syndrome du survivant"; "Il faut repenser au complet l'organisation du travail»; 
«Contre le chômage: le libéralisme "; "Hors du marché du travail, point de salut $" 1$.

On aura reconnu, à travers le martèlement de ces titres, les principales caractéristiques de l'évolution récente de l'emploi; je me contenterai de les énumérer et de les illustrer, à l'occasion, brièvement. La tertiariation de l'emploi, c'est-à-dire le passage de l'épicentre des activités économiques du secteur des ressources naturelles et de celui de l'industrie à celui des services et du commerce, s'est encore accélérée avec tous les bouleversements que ce déplacement suppose. Mais il y a plus; il faut surtout parler de la rarefaction ${ }^{2}$ croissante de l'emploi. Nous ne sommes plus en contexte de rareté des biens mais de surproduction ${ }^{3}$ et donc d'insuffisance de la demande. Cette surcapacité est évidemment éliminatrice d'emplois. Plusieurs facteurs concourent à ce déséquilibre. Ainsi une compétition, toujours plus ouverte et maintenant mondialisée, fait adopter des stratégies de survie et de conquête des marchés par les entreprises. Celles-ci sont poussées à réaliser des gains de productivité accélérés grâce aux " progrès " technologiques et à une "rationalisation" incessante des processus de production. D'où l'expulsion massive d'une main-d'œuvre qui devient "de trop ", "inutile », au point que le travail "exploité » en vienne à lui paraître enviable!

La Banque mondiale elle même reconnaît que "les décisions concernant les salariés et les conditions de travail sont dictées par des pressions compétitives mondiales ${ }^{4}$. Alors, un peu partout dans le monde, le chômage monte en flèche. Officiellement, le nombre global de chômeurs

1 Respectivement : Le Devoir, 27 janvier 1994; La Presse, 22 février 1994; Ibid., 30 juin 1995; Ibid., 14 février 1994; Le Devoir, 23 mars 1993; La Presse, 17 mars 1994; Le Devoir, 30 septembre 1993, Ibid., 15-16 janvier 1994; Ibid., 30 octobre 1994; La Presse, 11 mars 1994; Ibid. , 17 avril 1993; Le Devoir, (1989), citant le Conseil économique du Canada, L'emploi au futur (période :1980-1988); Le Devoir, 19 février 1990; Ibid., 8 août 1995; La Presse, 9 septembre 1994; Ibid., 5 juin 1992; Les Diplomés, automne 1995; La Presse, 4 janvier 1995; Le Devoir, 8 juin 1994; Ibid, 26 septembre 1995.

2 Voir, à ce sujet: M. BEAUDN, G. PAIEMENT (dir.), Sans emploi, peut-on viure?, (Défis de société), Montréal, Fides, 150 p.

3 N'est-il pas curieux qu'on s'acharne à accuser les gens d'avoir "vécu au-dessus de leurs moyens ", alors qu'on est en pleine crise de surabondance?

4 James D. WOLFENSOHN, préface à Le monde du travail dans une économie sans frontière. New York, Oxford Universiry Press, 1995 ciré dans J. DECORNOY, "Travail, capital... Pour qui chantent les lendemains?», Le Monde diplomatique, septembre 1995, p.18. 
atteignait 32,3 millions dans les pays les plus riches en 1992, selon 1'OIT ${ }^{5}$. Au Canada, le taux de chômage est de 9,6 \% (10,9\% au Québec). 71200 Canadiens (240 060 Québécois) ont reçu des prestations d'assurancechômage en mai 1995, une baisse de 40,9\% par rapport aux 1,2 millions de $1992^{6}$. Amélioration de l'emploi? Pas vraiment; il était plus simple d'éliminer des prestataires en resserrant les conditions d'accès au programme! Mais le taux d'inactivité est encore plus révélateur. Il "avoisinerait les $40 \%$, selon l'économiste Kimon Valaskakis ", "si l'on ajoute les personnes qui, découragées, ont cessé de chercher un emploi, celles qui vivent de l'aide sociale, les retraités «qui n'ont pas eu le choix» et ceux qui poursuivent leurs études parce qu'ils ne parviennent pas à décrocher un emploi ${ }^{7}$ ". Précisons aussi que le Québec comptait près de 800000 personnes assistées sociales au printemps 1995.

Pour illustrer la raréfaction de l'emploi en rapport avec l'ouverture des frontières économiques, on peut se référer aux conséquences de l'Accord de libre-échange avec les États-Unis entré en vigueur le ler janvier 1989. En 1985, la Commission Macdonald prévoyait la création de 650000 emplois dans les cinq ans suivant l'adoption de l'Accord. En 1987, le Conseil économique du Canada ramenait le pronostic à 350 000, alors que les opposants prédisaient une perte de 100000 emplois. Au début de 1988, le ministre des Finances, Michael Wilson, ne prévoyait plus que 120000 emplois créés. En décembre 1993, les cinq ans écoulés, le Canada avait déjà perdu 782000 emplois dont 407000 dans le secteur manufacturier (respectivement 257000 et 85000 pour le Québec).

Que penser de cette rareté croissante de l'emploi? Que l'humanité ait besoin de moins de travail humain pour se développer pourrait n'avoir rien de dramatique, au contraire. Ce serait même une libération, à condi-

5 «Monde du travail : dérapage incontrôlé », Le Devoir, 23 mars 1993.

6 "C'est au Québec qu'on trouve le plus grand nombre de prestataires de l'assurance- chômage ", La Presse, 27 juiller 1995.

7 Lia LÉVESQUE, "Le taux d'inactivité frôle les $40 \%$ selon l'économiste Valaskakis », La Presse, 11 juillet 1995. Certaines catégories de personnes sont plus durement touchées, ainsi les femmes, les immigrants et les jeunes. Dans ce dernier cas, le taux de chômage atteint le double de la moyenne des autres catégories d'âge : c'est structurellement que notre économie écarte les jeunes. «Le taux de participation des jeunes au travail actif est tombé de $70 \%$ en 1990 à $62,6 \%$ en 1995 », selon un directeur du Conference Board ( La concurrence et l'état du marché du travail ont nui à la reprise ", Le Devoir, 5 octobre 1995). 
tion, toutefois, que le revenu d'emploi tel que nous le connaissons cesse d'être le passage obligé pour la survie et le mieux-être.

Il faut encore parler, aujourd'hui, de dualisation du marché du travail et de précarisation de l'emploi. L'impératif de la compétitivité tend à créer deux "classes" de salariés. La performance exige, d'une part, un personnel très qualifié, première "ressource" de l'entreprise (la personne n'y reste encore qu'un moyen et non une fin de l'activité économique), et qui sera payé en conséquence. Les aléas du marché poussent, d'autre part, les entreprises à se délester de certaines rigidités (nombre fixe d'employés) et de certains coûts (avantages sociaux des employés réguliers), d'où la précarisation des autres emplois qui permet d'acheter le travail à la pièce selon les besoins. Cette "flexibilité " exigée des salariés s'appelle "précarité » chez les salariés: travail à temps partiel, à la pige, sur appel, saisonnier et en sous-traitance, par exemple. Comme me le disait avec tristesse une jeune jociste: "à vivre à côté du téléphone, tu ne fais pas de projets d'avenir. Les jeunes en sont réduits à des rêves cheap, limités aux préoccupations de logement, d'habillement, etc. ». Enfin, pour les gouvernements chargés de plier la main-d'œuvre aux besoins du capital, la même réalité se nomme "employabilité ". C'est la main-d'œuvre de ce second voler, la plupart du temps sous-utilisée et sous-payée, qui tend à devenir majoritaire. Elle est, en fait, condamnée aux "portes tournantes " du " bien-être social - programmes gouvernementaux de réinsertion au travail - jobbines - chômage (parfois, si éligible) - et à nouveau bien-être social, etc. ". C'est surtout dans ce sous-monde du travail que se fait la fameuse "création d'emplois ${ }^{8}$ "! C'est donc sans surprise que ce processus de dualisation et de précarisation du travail produit une société à deux vitesses, comme on le verra plus loin.

De tout ce qui précède surgit un autre trait: la "tiersmondialisation" des conditions de travail. À cause du mécanisme de concurrence internationale laissé à son libre jeu, à cause de la possibilité pour les entreprises d'aller s'établir là où les coûts de production sont les plus faibles ainsi que de la surabondance de la main-d'œuvre partout, il se produit une tendance à un nivellement par le bas qui nous rapproche des conditions de travail et de vie du Tiers-Monde. Le grand économiste américain Lester

8 Depuis 1980 , au moins $50 \%$ des nouveaux emplois sont de type précaire. Entre 1976 et 1994, le nombre de Québécois employés à temps partiel a bondi de 22600 à 51700 . De ces derniers, $42 \%$ le sont contre leur gré, alors que presque tous " avaient opté pour ce statut par choix ", il y a vingt ans (K. YAKABUSKI, "Travailler moins? Pourquoi pas! », Le Devoir, 11 juillet 1995). 
Thurow n'en fait pas mystère: "Aujourd'hui, pour être concurrentiel, un pays n'a que deux choses à offrir: des salaires plus bas et une maind'œuvre plus qualifiée. Il n'y a rien d'autre. " La dynamique est telle que même si la productivité des travailleurs augmente, ainsi de $30 \%$ aux ÉtatsUnis entre 1977 et 1992, le salaire moyen réel a chuté de $13 \%$ dans le présent cas $^{9}$. Que ne feraient pas les salariés pour conserver leur emploi? Et pourtant, rien n'est garanti. On ne peut plus parler de "nos" entreprises dans un contexte de mondialisation de l'économie. Le président de la filiale canadienne de la compagnie Northern Telecom (canadienne), M. R. Ferchat, disait en 1987: "Le développement de la compagnie n'est pas envisagé sur une base canadienne, ni même nordaméricaine, mais sur une base mondiale...la compagnie décidera du lieu de ses nouvelles usines en fonction de la proximité des marchés, de la disponibilité et du coût de la main-d'œuvre ${ }^{10}$ ». Le "chantage référendaire " encore plus récent de $M$. Laurent Beaudoin, président de la compagnie Bombardier qui doit sa prodigieuse expansion à l'appui financier de la population québécoise, confirme encore cette perspective.

Un dernier trait, celui de la dégradation du travail, découle directement du précédent. Les pressions du marché ont fait augmenter les heures et la charge de travail pour les employés réguliers, et fait se dégrader les conditions dans lesquelles le travail est exercé. Des recherches lient maintenant plus directement les problèmes de santé mentale et d'épuisement professionnel à ces changements.

Resaisissons ces données et leur impact social de manière plus synthétique à travers quelques paradoxes. Quelques titres de journaux serviront encore de point de départ: "L'économie va bien, mais les Canadiens s'appauvrissent "; "La reprise se fait sans emploi »; "La compéritivité au prix du chômage?"; "Youpi, le chômage monte "; "Le taux de chômage augmente, et les économistes croient qu'il s'agit d'un signe de reprise au

9 Michael LND, "To have or have not ". Notes on the progress of the American class war ", Harper's Magazine, juin 1995, cité par J. DECORNOY, loc. cit., p.18.

$10 \mathrm{M}$. TREMBLAY, «Northern Telecom Canada ne serait pas affectée par le libreéchange ", La Presse, 1 décembre 1987. Plus récemment, le président de la Banque de Montréal déclarait « qu'il ne voulait pas qu'on voit la Banque de Montréal comme une institution canadienne faisant quelques affaires aux États-Unis, mais bien comme une banque nord-américaine ayant des marchés des deux côtés de la frontière. "Notre but est de faire en sorte que cette frontière ne représente plus qu'une ligne imaginaire" ". (M. TISON, "La Banque de Montréal se voit comme une banque "nord-américaine" ", La Presse, 28 octobre 1994). 
Canada "; "Qui a peur de la croissance? Mais les financiers, voyons! "; "Rompre le couple expansion-exclusion" 11 .

Ces titres ne peuvent être plus expressifs; ils reflétent des statistiques accessibles à tous. Ainsi de 1983 à 1989, le monde occidental a connu une croissance sans précédent dans l'histoire; la situation de l'emploi n'a cependant cessé de s'y dégrader. Qui a donc confisqué cette richesse? Nous assistons à un phénomène inédit, à une "révolution " que me confirmaient, en entrevue, même de hauts dirigeants d'affaires francophones de Montréal: la restructuration néo-libérale signifie la simultaneité de la croissance et du chômage et donc de la pauvreté. C'est un euphémisme: je dirais plutôt que l'économie va bien parce qu'il y a plus de chômage et que les gens vont plus mal. Le chômage apparaît désormais comme un mal inhérent au fonctionnement du système. L'ambassadeur d'Allemagne à Rome, M. Konrad Seitz, ne cachait pas le "prix » d'une "restauration de la compétitivité»: "une réduction massive de la force de travail, une augmentation massive du chômage ${ }^{12}$ ".

C'est donc stucturellement que la croissance économique et l'emploi ont été découplés. Le travail est désormais considéré comme un poids pour l'économie, comme une tare et une souillure dont il faut débarrasser l'appareil productif. La main-d'œuvre en est venue à être considérée comme la principale cause des difficultés de «notre " économie, nos produits et services s'avérant trop chers à cause des salaires. Il y a là un beau prétexte à faire reporter toutes les contraintes du côté de la population.

Une nouvelle, devenue courante, comme la suivante, illustre cruellement notre paradoxe: à la fin de 1989 , après une réduction de ses effectifs de 25000 personnes, la compagnie ATT annonçait la suppression de 8500 autres emplois pour 1990, en raison de la "mise en place d'une technologie plus avancée " et de la "vive concurrence ». Cette annonce, nous dit-on, « a entraîné une hausse du titre ATT qui gagnait 37,5 cents... à Wall Street ${ }^{13}$ "! La logique économique, et financière surtout, semble s'être tout à fait détachée de la téalité sociale.

11 Respectivement : Le Devoir, 21 avril 1988; La Presse, 14 mai 1994; Le Devoir, 23 avril 1993; La Presse, 12 mai 1994; Ibid., 6 juin 1992; Le Devoir, 4 mars 1994; Le Monde, 30 décembre 1994.

12 Propos cité dans F.F. ClAIRMONT, « La diabolique logique de la productivité », Le Monde diplomatique 484 (juillet 1994), p.18, et reproduits de International Herald Tribune, 10 mars 1994.

13 «ATT supprime 8500 emplois», Le Devoir, 12 décembre 1989. 
L'économie néo-libérale détruit les emplois, mais ne relâche pas son exigence inflexible d'un revenu... d'emploi! Il s'en suit un appauvrissement marqué de la population et un phénomène massif d'exclusion sociale. La société se casse en deux: on voit de plus en plus de Jaguars et ... de sans-abri dans les rues! Aux États-Unis, cas extrême, " les $1 \%$ de foyers les plus riches possèdent presque $40 \%$ de la richesse nationale ${ }^{14}$ ". Au Canada, 2 millions de personnes doivent avoir recours à l'aide alimentaire au moins une fois l'an ${ }^{15}$. Chez-nous, 500 organismes d'aide alimentaire quadrillent maintenant le Montréal Métropolitain ${ }^{16 !}$ Les sociétés se disloquent et des couches entières de population "s'installent dans la précarité ". Le néo-libéralisme crée un "déficit structurel des places occupables dans la sociétét ${ }^{17}$ ". Et quelle solution offre-t-il face à ce problème: à côté de certains patrons allemands qui proposent de "moins travailler " comme "objectif central de la société allemande", ceux des États-Unis avancent plutôt l' «allongement de la semaine de travai118 " et surtout aucun "salut " "hors du marché du travail 19 », dûton n'avoir rien d'autre à offrir que des "Mc jobs". "Tout travail vaut mieux que pas de travail », avait un jour lancé l'ancien ministre de la Main-d'œuvre et de la Sécurité du revenu, M. André Bourbeau. Au fond, le capitalime nord-américain n'a pas d'autre remède à proposer que la cause même du mal ${ }^{20}$. À ce sujet, je renvoie le lecteur à l'article d'André Gorz (pages 73-88).

Le travail se trouve donc coincé, de nos jours, entre les aspirations et nécessités humaines auxquelles il répond de plus en plus mal, et une économie devenue folle qui l'asservit à des fins qui nous paraissent de plus en plus étrangères. Pour mieux « déchiffrer " la logique qui préside à ce

14 K. BRASDHER, "U.S. Ranks First in Economic Inequality ", International Herald Tribune, 18 avril 1995, cité dans J. DECORNOY, loc. cit. p. 18.

15 Statistiques Canada, Tendances sociales canadiennes, 1992.

16 J. PICHETTE, « Une société underground », Le Devoir, 17 mai 1995.

17 Sur la nouvelle question sociale posée par cette dynamique et sur sa mise en perspecrive historique, voir R. CASTEL, Les métamorphoses de la question sociale. Une chronique du salariat. (L'espace du politique), Paris, Fayard, 1995, 490 p., et P. Rosanvallon, La nouvelle question sociale. Repenser l'Etat-providence. Paris, Seuil, $1995,223 \mathrm{p}$.

18 K. YAKABUSKI, loc. cit., cirant J. RIFKIN, The End of Work, 1995.

19 G. FRASER, « Hors du marché du travail, point de salut », Le Devoir, 26 septembre 1995.

20 J.-M. BELOT, « Contre le chômage : le libéralisme », Le Devoir, 8 juin 1994. 
paradoxe persistant, il nous faut faire un peu d'histoire et d'analyse économique. Il nous faut remonter jusqu'au début du siècle et considérer les profils structurels (ou modèles) successifs du capitalime, qui ont été, à chaque époque, absolument déterminants pour la réalité du travail. Nous serons mieux en mesure alors de cerner la genèse de néo-libéralisme actuel et le défi qu'y rencontre le travail humain. Pour mieux mettre en évidence la logique qui a dominé à chaque étape de cette trajectoire, je négligerai les expressions d'une résistance continue à celle-ci ${ }^{21}$.

\section{Le travail au gré des soubresauts du capitalisme au $\mathrm{XX}^{\mathbf{e}}$ siècle}

Trois modèles d'économie capitaliste ont scandé le $\mathrm{XX}^{\mathrm{e}}$ siècle ${ }^{22}$. Le premier, commencé au XVIII e siècle, s'est épuisé avec la crise des années 30. Le second, appelé fordo-keynésien, s'est mis peu à peu en place à la fin des années 30, pour s'épanouir jusqu'au milieu des années 60 et s'estomper par la suite. Enfin, après une décennie de tâtonnements, le modèle néo-libéral prendra la relève, des années 80 à nos jours où il semble toujours triompher.

\subsection{Jusqu'aux années 30: le libéralisme "sauvage»}

Le capitalisme ou libéralisme "pur» du début du $\mathrm{XX}^{\mathrm{e}}$ siècle, comme celui du XIX $\mathrm{X}^{\mathrm{e}}$ siècle, base la progression des profits sur l'exploitation maximale de la main d'œuvre. Il oppose carrément le capital et le travail. Il est "labour intensive », en raison d'une technologie relativement peu développée. Pas question, ici, d'une croissance axée sur la demande locale, d'ailleurs quasi inexistante avec de si maigres salaires. C'est une économie de l'offre. Chômage, travail précaire et recours à l'économie de subsistance (comme aujourd'hui dans le Tiers-Monde) sont le lot des ouvriers. D'où la condition misérable de ceux-ci " isolés et sans défense... livrés à la merci de maîtres inhumains et à la cupidité d'une concurence effrènée ", selon les termes mêmes de Léon XIII. " À tout cela, il faut ajouter, dit-il, la concentration entre les mains de quelques-uns, de l'industrie et du commerce, devenus le partage d'un petit nombre de riches et d'opulents, qui imposent un joug presque servile à l'infinie multitude des

21 Pour les efforts de recomposition sociale, à partir d'initiatives communautaires souvent mal connues, je renvoie, par exemple, à : M. NOzıck, Entre nous. Rebâtir nos communautés. Montréal, Éditions Ecosociété, 1995, 264 p.

22 Voir le tableau-synthèse à la page 32. 
prolétaires 23 ". On a comparé les conditions de vie du Montréal de cette époque à celles de Calcutta.

En conséquence des principes du "laisser-faire ", et de la prétendue capacité autorégulatrice du marché imposés depuis le siècle passé, l'État n'intervient pas. Les politiques économiques restent confinées aux dépenses militaires, de police, etc., et les politiques sociales sont inexistantes si on excepte un appui minimal à quelques institutions de charité. On connaît la suite. Le développement technologique, combiné à la faiblessse de la demande solvable, provoque une surproduction et un stockage qui inciteront tous les propriétaires d'actions à vouloir vendre en même temps en 1929: c'est le crash, consacrant le déséquilibre d'une économie d'exploitation, suivi de la grande dépression des années 30 .

Des observateurs ont souligné, avec raison, la similitude de la situation actuelle avec celle de cette époque. Le néo-libéralisme fait, en effet, retourner le travail à la norme séculaire du capitalisme: la précarité. Écoutons Pie XI, cette fois, en 1931: "Une loi économique inéluctable, assurait-on, voulait que tout le capital s'accumulât entre les mains des riches; la même loi condamnait les ouvriers à traîner la plus précaire des existences dans un perpétuel dénuement ${ }^{24}$. »

\subsection{Des années 40 à la fin des années 60 :}

le modèle fordo-keynésien

Pour débloquer le retour des profits, on propose un "New Deal " (1936) aux populations. À la suite de Henry Ford, les industriels misent sur une demande ou consommation de masse croissante, capable de suivre le pas d'une production de masse que le progrès technologique rend possible. Vu le caractère essentiellement national de l'économie, le succès de l'offre rend obligatoires l'emploi généralisé et le relèvement des salaires. La guerre aidant, l'économie repart, le chômage se résorbe (1,8\% en 1948) et les profits reviennent.

Sous l'inspiration de l'économiste anglais John M. Keynes, l'État est aussi mis à contribution comme régulateur de la demande. Il assumera ce rôle par la mise en place de politiques sociales (allocation familiale, assurance-chômage...) qui assurent une utilité économique même aux mères de famille à la maison ou aux chômeurs (ou "bras inutiles") précisément

Rerum novarum, $\mathrm{n} 2$

24 Quadragesimo anno, n. 60. 
comme « consommateurs 25 ", et par l'intermédiaire de politiques monétaires (taux d'intérêts) et fiscales qui font augmenter ou baisser, au besoin, les revenus de consommation des gens, permettant ainsi de corriger une inflation ou un chômage excessifs. On appellera keynésianisme ce rôle économique (et non d'abord humanitaire) de l'État soutenant indirectement l'offre (production et profits) par la demande. Il en résultera la grande prospérité et le système de sécurité sociale de l'après-guerre, une heureuse parenthèse dans la trajectoire historique d'un capitalisme prenant à ce moment-là un visage plus humain dans les pays riches. Dans ce modèle, la croissance passait par une alliance avec le travail ${ }^{26}$ et par le bien-être des populations. Il marqua l'apothéose de la "société salariale $»$.

Mais vers le milieu des années 60 , ce modèle s'épuise pour diverses raisons, cessant d'assurer des taux de profit croissants. Le marché local se réduit maintenant au seul remplacement des équipements usuels (autos, appareils ménagers...); les pays du Tiers-Monde revendiquent des prix plus justes pour leurs matières premières (le pétrole est encore au prix de 1907); les équipements industriels ont vieilli et sont moins efficaces; et, enfin, les économies européenne et japonnaise, reconstruites à neuf, offrent des produits à prix plus compétitifs et gagnent rapidement du terrain sur les marchés aux dépens de l'Amérique du Nord.

\subsection{Des années 70 à nos jours: le modèle néo-libéral}

En réaction, un nouveau remodelage du capitalisme se dessinera peu à peu, dans les années 70, à partir d'initiatives des grandes entreprises et des banques. On force les profits tant du côté de l'offre que de la demande.

Dans le premier cas, le recouvrement des profits passe par une compression des coûts de production: mises à pied, restrictions salariales, détérioration des conditions de travail, précarisation de l'emploi, santé et formation de la main d'œuvre aux frais de la collectivité (système d'éducation et assurance-santé), nouvelle technologie, restructuration (acquisitions et fusions d'entreprises, fermetures d'usines...),

25 C'est le nom que prennent désormais les citoyennes et les citoyens désignés ainsi selon leur nouvelle fonction dans le système économique. "Born to shop », aurait-on pu écrire sur le berceau de chacune et de chacun.

26 Au début des années 60 , le taux de chômage "normal " est sensé ne pas dépasser $3 \%$. 
"rationnalisation" des processus de production et de la gestion, déplacement de la production vers des régions plus "accueillantes " (avec chantage aux subventions), etc. C'est la revanche du capital sur le travail et sur ses acquis des décennies précédentes. Du côté de la demande, le "salut ", attendu d'abord d'une hausse des prix (inflation), se rabat ensuite sur la conquête des marchés internationaux et sur l'élimination des concurrents plus faibles. L'offre et la demande sortent du cadre national et des protections correspondantes pour se donner le monde entier en "pâturage ".

Les économistes F.A. Hayek (Vienne) et M. Friedman (École de Chicago) théoriseront cette approche dans le modèle néo-libéral qui sera adopté d'abord par les gouvernements Reagan, Thatcher, après son expérimentation au Chili de Pinochet (1973-), puis Mulroney et Bourassa chez nous. Réquisitionné ou "satellisé ", l'État se fait complice de la course à la "compétitivité ". Il ne s'agit plus de "laisser faire", mais de "faire aller »! Cela se traduit par des «faveurs" pour attirer des investissements, par des politiques libre-échangistes, par des dégrèvements fiscaux 27 , par des politiques monétaires aberrantes 28 , et par une compression des programmes sociaux qui a pour effet de forcer les sansemploi à s'offrir comme main-d'œuvre à n'importe quel prix et ceux qui ont déjà un emploi à se faire moins exigeants, favorisant ainsi une baisse des coûts de production. Et qu'importe si les gens ont moins de revenus puisque le profit passe désormais moins par le marché local que par les exportations et la production à l'étranger et surtout les activités financières 29 .

27 Comble de l'absurde, des impôts des salariés servent toujours à subventionner des entreprises pour l'acquisition de nouvelles technologies qui justifieront leur propre mise à pied.

28 Les pays ne sont plus dirigés mais "gérés ». C'est le FMI (programmes d'ajustement structurel) et les intérêts des détenteurs de capitaux qui dictent la politique canadienne. Pour préserver les gains de ces derniers et attirer les prêts, le gouvernement a donné priorité à la lutte contre l'inflation par des taux d'intérêts insensés qui ont ralenti l'économie, provoqué du chômage et accrû ses déficits et sa detre. Statistiques Canada (1991) a reconnu que seulement 6\% de l'augmentation de la dette canadienne depuis 1975 pouvait être imputé aux programmes sociaux contre $50 \%$ et $44 \%$ respectivement à la hausse des taux d'intérêts et aux abris fiscaux.

29 Selon $N$. Chomsky, les échanges de capitaux internationaux sont maintenant liés à $90 \%$ à la spéculation financière plutôt qu'à la production comme il y a vingt ans, et le développement des télécommunications leur permet d'échapper à tout contrôle public et fiscal (A. NOEL, "Chomsky dénonce le grand capital, la globalisation et les médias », La Presse, 8 octobre 1995). 
Les «bras inutiles" (chômeurs, personnes assistées sociales...) deviennent aussi des "bouches inutiles"! Fini donc l'État-providence, les gouvernements ont autre chose à faire! On lui substitue l'État-business assorti d'un discours sur la responsabilité et la solidarité des citoyens, renvoyant ainsi les appauvris à la charité publique spontanée, que celle-ci prenne la forme du bénévolat, de téléthons, de Centraide ou d' " œuvres " d'Église!

Dès 1931, Pie XI avait déjà anticipé la logique néo-libérale. Après avoir dénoncé la "dictature économique " des grands monopoles qui rendait "toute la vie économique horriblement dure, implacable et cruelle ", il décrit ainsi la collusion des États:

À tout cela viennent s'ajouter les graves dommages qui résultent d'une fâcheuse confusion entre les fonctions et devoirs d'ordre politique et ceux d'ordre économique: pour n'en citer qu'une d'une extême importance, la déchéance du pouvoir: lui qui devrait gouverner de haut, comme souverain et suprême arbitre, en toute impartialité et dans le seul intérêt du bien commun et de la justice, il est tombé au rang d'esclave et devenu le docile instrument de toutes les passions et de toutes les ambitions de l'intérêt ${ }^{30}$.

Voilà le modèle qui est proposé comme voie d'avenir, mais qui ne marque qu'un retour au vieux libéralisme doublé, cette fois, d'un soutien encore plus direct de l'État (" néo-") et des ressources publiques. Voici comment Michael Porter, un grand "gourou " de l'économie néo-libérale, décrit le rôle attendu des États: « les gouvemements doivent fournir aux entreprises nationales des conditions propices à leur croissance sans les protéger des innovations et de la concurrence des marchés qui s'orientent vers la mondialisation 31 \%. On devine sans mal qui fera les frais de cette compétition ouverte.

J'ai déjà décrit plus haut la tyrannie exercée sur le travail et les conditions de vie des populations par la dynamique néo-libérale. Cellesci sont prises en otage par la guerre que se livrent les nouveaux seigneurs féodaux. Pour survivre, elles doivent consentir à une mobilisation générale de toutes les composantes de la société en faveur du petit nombre, et avec cette triste perspective, en cas de réussite, de devoir faire des perdants

30 Quadragesimo anno, n.117.

31 The Competitive Advantage of Nations, New York, Free Press, 1990, cité dans “Une étude d'un « gourou » américain influencerait le débat sur l'autonomie », La Presse, 17 mars 1991. 
dans les autres populations du globe, avec lesquelles elles sont involontairement mises en concurrence, comme l'illustre l'article de Carlos Heredia (pages 33-44) à propos du Mexique. Le néo-libéralisme nous enferme dans une logique de guerre. Un indice de la fausseté de ce jeu funeste: jamais n'aura-t-on connu autant d'exclusion sociale (individus, quartiers, régions et même continents entiers) que depuis qu'on parle d'ouverture, de "progrès " et d'universalisme pour qualifier la compétition internationale. Le néo-libéralisme marque l'exaspération de l'économie de marché et peut-être aussi sa fragilisation décisive, du moins celle de la "société salariale".

Que faire quand l'anti-solidarité s'inscrit dans la fibre même de l'organisation économique qui disloque la société et qui réduit les gens au statut de "quêteux d'emploi prêts-à-jeter », quand l'économie trahit la vocation indiquée par son étymologie - oikos (maison), nomos (gestion) soit d'être gestion de la société comme une maisonnée fraternelle où personne ne soit "de trop ", et non comme une jungle? Comment échapper à la culture du désespoir qui s'infiltre par la perversion du travail humain?

\section{Le travail sous la coupe de l'economically correct}

Ce que je viens d'esquisser ressemble fort à une impasse, mais celle-ci est-elle insurmontable? L'espoir me semble passer par la prise de conscience de la relativité de ce que le néo-libéralisme tend à faire croire comme une loi intouchable, comme une fatalité à laquelle la sagesse commanderait de s'adapter. C'est ce que je voudrais aborder en dernier lieu par la dialectique que j'appellerais de l'illimité et de la limite, et qui me paraît concerner à la fois la dynamique concrète et l'idéologie du néo-libéralisme.

\subsection{L'illimité ou l'infinitude perverse}

Le néo-libéralisme a porté à son plus haut degré le processus d'autotranscendance ou de «sumaturalisation » du paradigme du marché. Celuici semble, en effet, doué d'une autonomie et d'une extériorité par rapport aux humains qui n'est pas sans rappeler l'Ananke (nécessité) grecque ou le Fatum romain, et qui le fait échapper principiellement à toute transcendance et maîtrise humaine et sociale à son égard. Au plan idéologique (science économique), cela se traduit dans la croyance en l'économie de marché comme en un "ordre spontané " ou autorégulateur (Hayek), comme l' "état de nature " de l'économie, et dans une position anti-uto- 
pique qui fait considérer le capitalisme actuel comme la «fin de l'histoire" .

La sacralisation du marché comporte une sorte de revendication de son caractère illimité. Cette infinitude semble inscrite dans son fonctionnement même: les visées de progrès matériel, de l'enrichissement et du profit ne connaissent pas de limite, dans une humanité pourtant marquée par la finitude. La concurrence entre entreprises n'a pas d'autre point de repère que l'action des compétiteurs. Elle entraîne ceux-ci dans une spirale sans fin, à grossir indéfiniment et à toujours mieux se positionner stratégiquement ou à périr, sans parler des "tiers" qui feront les frais de cette lutte des "doubles" pour utiliser des catégories girardiennes.

Mais toute finitude injustifiée ne devient-elle pas perverse et infernale comme le démontre le processus de destruction sociale et environnementale qui accompagne la marche accélérée du capitalisme néo-libéral. Celui-ci est marqué par une logique close sur elle-même, par une autoréférentialité qu'on nomme idolâtrie en termes théologiques ${ }^{32}$. Prenons comme exemple le cycle désespérant engendré par la sacro-sainte compétitivité qui ne sert qu'à se nourrir elle-même sans fin dans un processus qui se double d'une vaste exclusion sociale. Ainsi les gains de productivité obtenus grâce aux efforts des populations et qui devraient normalement leur revenir sous formes de bien-être et de meilleurs services sociaux et culturels, par exemple, doivent plutôt être immédiatement réinvestis pour garder les entreprises compétitives et ainsi obtenir de nouveaux gains de productivité qui, à leur tour... etc, etc, etc. La logique de la concurrence sans frein et sans fin condamne à ne jamais toucher les fruits du travail. Et dans ce processus, combien de personnes auront perdu leur emploi ou l'auront vu se détériorer?

\section{2 "Limites" et sacrificialité imposées par le néo- libéralisme " 33}

L'autoréférentialité du néo-libéralisme comporte de sa part, comme corollaire, l'imposition de "limites " concernant à la fois le fontionnement économique possible et les valeurs (idéologie) admissibles. Je me

32 Voir à ce sujet mes deux arricles dans le dossier « Cette idole qui nous gouverne » dans Relations, octobre 1995.

33 Je suis surtout redevable, pour la conceptualisation de cette partie à Hugo ASSMANN, "Idolâtrie du marché et sacrifices humains", dans H. ASSMANN et F.J. HINKELAMMERT, L'idolâtrie du marché. Critique théologique de l'économie de matché. (Libération), Paris, Cerf, 1993. 185-243. 
limiterai ici à quelques exemples de la détermination de l'economically correct qui permet de "fonctionnaliser " à l'intérieur du système économique toute l'activité et même le sens de la vie humaine:

- le refus de considérer les besoins humains de base s'ils ne peuvent se traduire en demande solvable;

- le refus d'accorder quelque valeur à ce qui ne peut s'acheter ou se vendre et la mobilisation de toutes les énergies humaines vers cet objectif exclusif 34 ;

- la croissance (et non le développement) indéfinie comme seule solution aux problèmes économiques et sociaux issus de la croissance mêrne;

- la vision tronquée de la réalité imposée par les indicateurs et statistiques économiques;

- la rentabilité comme condition ou critère unique de mise en œuvre d'un projet;

- l'obligation de "rassurer " d'abord les marchés financiers plutôt que les populations, par des politiques kafkaiennes qui détachent la sphère financière de toute logique économique et sociale;

- le respect absolu des contrats malgré les coûts humains et sociaux, comme l'illustre l'exigence inflexible du remboursement de la dette du Tiers-Monde pourtant impayable, et source d'un holocauste silencieux;

- la considération du travail humain qu'à l'intérieur de la clôture de l' « emploi ";

- la mobilité imposée à la main-d'œuvre à l'exemple du capital;

- le déplacement à la hausse de la "normalité " du taux de chômage, maintenant fixé à $10 \%$;

- la capacité idéologique de faire croire que la création d'emplois relève d'abord des gouvernements et de leur faire porter l'odieux de la situation. Qui n'a pas fait sa «job", l'entreprise ou l'Étrat?

On aura facilement saisi que c'est constitutivement que l'economically correct du néo-libéralisme entraîne le sacrifice des plus faibles. Une fois

34 E. KITZMOLleR, “Economy as a Victimizing Mechanism», Contagion 2 (printemps 1995), p. 20-21. 
incorporés à la logique du capital comme "travailleurs", "consommateurs ", "contribuables ", "clients ", ou «bénéficiaires ", etc., les citoyens et même toute la société deviennent potentiellement sacrificiables. La légitimation sacrificielle ne s'appuie plus sur une réalité extérieure comme traditionnellement, mais elle «s'identifie entièrement à la formulation des mécanismes économiques 35 ».

Dans un ouvrage récent, un journaliste économique, P. Thureau-Dangin, se demandait si on n'avait pas fait assumer aujourd'hui à la concurrence le rôle joué traditionnellement par la mort comme aiguillon de nos existences, en réduisant ces demières à la seule dimension de la lutte économique et en banalisant leur sacrifice ${ }^{36}$.

La socialisation et la légitimation du "jeu " de la concurrence dédouanent du même coup ses résultats: les inégalités structurelles. Et c'est ainsi que de victimes et sacrifiés, les chômeurs, les chômeuses et tous les pauvres deviennent coupables de leur sort et, conséquemment, seuls responsables de leur propre relèvement. Aux États-Unis, en Alberta, en Ontario, et peut-être ici, demain, on passe de la compassion, à l'indifférence puis à la culpabilisation des exclus de l'emploi. Le capitalisme se fait maintenant plus ouvertement cynique; on n'est plus très loin de l'affirmation nietzschéenne des nécessaires sacrifices humains pour le bien de toute la société $e^{37}$.

Le modèle néo-libéral bloque graduellement à la majorité la voie principale du "gagne pain» : l'emploi. Mais les gouvernements néo-libéraux sont aussi en train de rétrécir et même de murer la sortie de secours: les prestations d'aide sociale de toutes sortes. La population se trouve structurellement prise au piège comme l'illustre bien un vote récent sur l'aide sociale, au Sénat américain, qui rompt avec le «New Deal». On déclare la "guerre aux pauvres". "En définissant l'aide sociale comme

35 Hugo AsSMANN, op. cit., p.197.

36 La concurrence et la mort. Paris, Syros, 1995, 215p.

37 «L'individu a été si bien pris au sérieux, si bien posé comme un absolu par le christianisme, qu'on ne pouvait plus le sacrifier : mais l'espèce ne survit que grâce aux sacrifices humains...La véritable philanthropie exige le sacrifice pour le bien de l'espèce; elle est dure, elle oblige à se dominer soi-même, parce qu'elle a besoin du sacrifice humain. Et cetre pseudo- humanité qui s'intitule christianisme veur précisément imposer que personne ne soit sacrifié... (CEuvres complètes, XIV. Fragments posthumes 88-89, Paris, Gallimard, 1977, p. 224-225, cité dans R. GIRARD, Quand ces choses commenceront... Entretiens avec Michel Treguer. Paris, Arléa, 1994, p. 19 (les italiques sont de René Girard). 
une cause de la pauvreté, on justifie son élimination...les pauvres sont corrompus par l'aide sociale, ils seront purifiés par les compressions ${ }^{38}$ ». Et on leur indique ensuite la seule voie possible: la stricte "limite» du paradigme néo-libéral, soit le marché du travail, mais pour quels emplois?

\section{Conclusion}

Cette brève esquisse de la figure contemporaine de la crise du travail pose des questions à la fois pratiques et théoriques aux citoyens et aux citoyennes. Saurons-nous reconstituer un sujet social, une société civile, elle seule en mesure, bien plus que des individus isolés, de reprendre la maîtrise du marché et de l'État afin, sans abolir ceux-ci, de les mettre au service de son propre agenda? Pouvons-nous envisager de sortir le travail du carcan exclusif de l'emploi et d'en institutionnaliser une finalité humainement et socialement acceptable? Mais la simple considération de ces perspectives ne suppose-t-elle pas une reconquête de notre imagination et de notre liberté d'esprit, peu à peu et imperceptiblement subjuguées par l'idéologie néo-libérale? Ne suppose-t-elle pas de comprendre que le travail est plus que le "travail"? Que l'économie est plus que l' «économie »? Et que leur configuration singulière actuelle reste historiquement relative, à l'encontre de toute fatalité? En somme, que la société n'est pas réductible au marché?

Enfin, peut-être les chrétiens et chrétiennes se seront-ils sentis interpellés par l'enfermement et par les "sacrifices humains" perpétrés au nom d'une prétendue orthodoxie, fût-elle économique? Le fondamentalisme économique qui occupe et structure le terrain public comme nos vies personnelles, nous transformant en pratiquants dociles de la "religion» marchande, est-il vraiment compatible avec le Dieu de la vie et avec son projet pour l'humanité? L'Évangile a-t-il été trop domestiqué pour nous inspirer encore une résistance face au totalitarisme du marché? La solidarité avec les victimes du néo-libéralisme et l'adoption de leur perspective ne seront certes pas superflues pour nous en faire retrouver la lucidité et pour "transcender" les limites frauduleusement imposées par un capitalisme qui n'a que des solutions du XVIII ${ }^{\mathrm{e}}$ siècle à proposer pour nous permettre d'affronter les défis du XXI ${ }^{e}$ siècle.

38 G. FRASER, " Hors du marché du travail, point de salut », Le Devoir, 26 septembre 1995. Voir aussi : S. BUREAU, « Le confort et l'indifférence ", La Presse, 25 mars 1991, et M. ROCHE, «Les Britanniques ne supportent plus leurs mendiants », Le Devoit, 7-8 octobre 1995. 


\section{Bibliographie}

BEAUDIN, Michel et Guy PAIEMENT (dir.), Sans emploi, peut-on viure? (Défis de sociéré), Montréal, Fides, 1994, $150 \mathrm{p}$.

BIDET, Jacques et Jacques TEXIER (dir.), La crise du travail. Paris, P.U.F., 1995, 264 p.

CASTEL, Robert, Les métamorphoses de la question sociale. Une chronique du salariat. (L'espace du politique), Paris, Fayard, 1995, 490 p.

CHENAIS, François, La mondialisation du capital. (Alternatives économiques), Paris, Syros, 1994, $286 \mathrm{p}$.

EME, Bernard et Jean-Louis LAVILLE (dir.), Cohésion sociale et emploi. (Sociologie économique), Paris, Desclée de Brouwer, 1994, 283 p.

GODINOT, Xavier (dir.), * On voudrait connaitre le secret du travail ^. (Des livres contre la misère), Paris, Éditions ATD Quart Monde, 1995, 352 p.

GORZ, André, Métamorphoses du travail. Quête du sens. Critique de la raison économique. (Débars), Paris, Éd. Galilée, 1991, 303 p.

HERZOG, Philippe (dir.), Changer d'économie. Paris, Syros, 1993.

JACQUART, Albert, J'accuse l'économie. Paris, Calmann-Lévy, 1995, 172 p.

LANGLOIS, Richard, Pour en finir avec l'économisme. (Pour en finir avec), Montéal, Boréal, 1995, $176 \mathrm{p}$.

MEDA, Dominique, Le travail. Une valeur en voie de disparition. (Alto), Paris, Aubier, 1995, $358 \mathrm{p}$.

PERRET, B. et G. ROUSTANG, L'économie contre la société. Affronter la crise de l'intégration sociale et culturelle. (Esprit), Paris, Seuil,1993, $284 \mathrm{p}$.

ROCAJQ, Les valeurs et les pratiques des jeunes face au travail et au non-travail. Fondation Jeunesse 2000, 420, rue Saint-Paul est, 3e étage, Montréal, Qué., H2Y $1 \mathrm{H} 4$.

ROSANVALLON, Pierre, La nouvelle question sociale. Repenser l'État-providence. Paris, Seuil, 1995, 223 p.

THUREAU-DANGIN, Philippe, La concurrence et la mort. Paris, Syros, 1995, 214 p.

TREMBLAY, Diane-Gabrielle, Economie du travail. Les réalités et les approches théoriques. Montréal, Éditions Saint-Martin et Télé-Université, 1990, 544 p.

Plusieurs numéros de la revue Relations (ex. mai 1992, janvier-février 1993, mai 1993, septembre $1994 \ldots$ )

Revue Virtualités, 853 Sherbrooke Est, Montréal, Qué., H2L 1K6. (ex. novembre 1993, mars-avril $1994 \ldots$ ) 
LE TRAVAIL AU GRÉ DES REMODELAGES DU CAPITALISME AU XXe SIÈCLE

\begin{tabular}{|c|c|c|c|c|c|}
\hline EPOQUE & ECONOMIE & $\begin{array}{l}\text { POLITIQUES } \\
\text { ECONOMIQUES }\end{array}$ & $\begin{array}{l}\text { POLITIQUES } \\
\text { SOCIALES }\end{array}$ & SOLIDARITÉ & IDÉOLOGIE \\
\hline $\begin{array}{c}\text { AVANT } \\
1935\end{array}$ & $\begin{array}{c}\text { CAPITAL vs TRAVAI } \\
\text { EXPLOTTATION MAX. } \\
1929: \text { O > D } \\
\text { = LIBERALISME "PUR" }\end{array}$ & $\begin{array}{c}\text { NULLES SAUF VIA } \\
\text { DEPEENSES MILTTAIRES }\end{array}$ & $\begin{array}{l}\text { NULLES OU PRESQUE } \\
\text { ETAT SUPPLETIF } \\
\text { ASSISTANCE PRIVEE }\end{array}$ & $\begin{array}{ll}\text { "CHARTE" + ENTRAIDE } \\
-\quad \text { LOCALE } \\
-\quad \text { FAMIIALE } \\
-\quad \text { INFORMELLE }\end{array}$ & $\begin{array}{l}\text { LIBRE MARCHE } \\
\text { AUTOREGULATEUR } \\
\text { (XIX' slècle) } \\
\text { «LAISSER-FAIRE» }\end{array}$ \\
\hline $\begin{array}{c}1935 \\
\text { d } \\
1968\end{array}$ & $\begin{array}{l}\text { CROISSANCE BASEE } \\
\text { SUR EQUILIBRE } \\
\text { O }=\text { D (en Spirale) } \\
\text { - NATIONALE SURTOUT } \\
\text { CHOMAGE "NORMAL" } \\
=3 \% \\
=\text { FORDISME }\end{array}$ & $\begin{array}{c}\text { SOUIIEN DE O } \\
\text { VIA SOUTIEN DE D } \\
\text { (impôts, \% d'intéréts etc.) } \\
\text { INTERVENTION DE } \\
\text { L'ETAT } \\
\text { = KEYNESIANISME }\end{array}$ & $\begin{array}{l}\text { SOUTIEN DE O via } \\
\text { SOUTIEN DE D par } \\
\text { les programmes sociaux } \\
\text { disponibilité / travail } \\
\text { SECURITE "SOCIALE" } \\
\text { IUBLLQUE } \\
\text { ETAT-PROVIDENCE }\end{array}$ & $\begin{array}{l}\text { INSTITUTIONNALISÉE } \\
\text { au plan PROVINCIAL } \\
\text { NATIONAL }\end{array}$ & $\begin{array}{c}\text { CAPITALISME } \\
\dot{a} \\
\text { VISAGE HUMAIN } \\
\text { (contróle politique } \\
\text { pour redistribution) }\end{array}$ \\
\hline DATE & $\begin{array}{c}\text { RESTRUCTURATION DE } \\
\text { L'OFFRE } \\
\text { TRANSNATIONALISATION } \\
\text { DE LA PRODUCTION (O) } \\
\text { FUSIONS, ACQUISITIONS } \\
\text { TECHNOLOGIE } \\
\text { CQNQUETE DES } \\
\text { MARCHES (D) } \\
\text { D = Demande mondialc } \\
\text { nationalc } \\
\text { EMPLOI : chômage normal } \\
\text { - 10\% } \\
\text { précarisation } \\
\text { - NEO-LJBERALISME } \\
\text { (OU Economic de l'OFFRE) }\end{array}$ & $\begin{array}{c}\text { REORIENTATION DU } \\
\text { ROLLE ECONOMIQUE DE } \\
\text { L'ETAT : } \\
\text { SOUTIEN DIRECT A O } \\
\text { (subventions, fiscalité ...) } \\
\\
\text { "PROVIDENCE DES } \\
\text { GROSSES ENTREPRISES" }\end{array}$ & $\begin{array}{l}\text { REOORIENTATION DES } \\
\text { POLTIQUES SOCIALES } \\
\text { - SOUTIEN A O par } \\
\text { l'incitation à l'emploi } \\
\text { - DEMANTĖLEMTNT } \\
\text { PROGRESSIF DES } \\
\text { PROGRAMMES } \\
\text { SOCIAUX } \\
\text { SECURITE "SOCIALI" } \\
\text { - SECURITE DU "RLVIENU" } \\
\text { ETAT INDIFFERENCE } \\
\text { OU "WORKFARE STATE" }\end{array}$ & 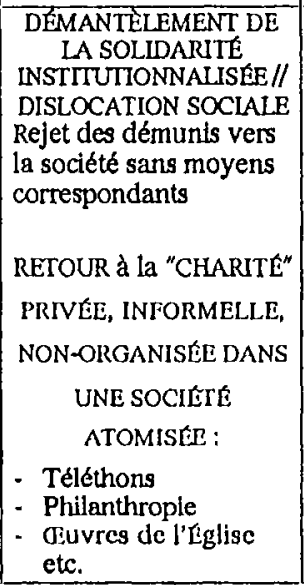 & 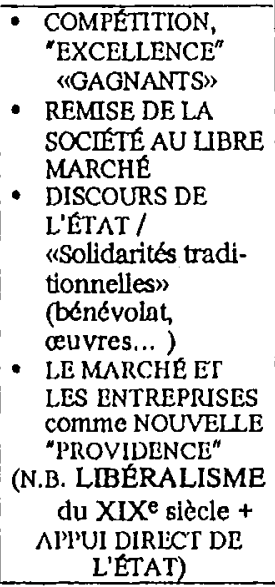 \\
\hline
\end{tabular}

